\title{
Titanate Nanotubes for Reinforcement of a Poly(Ethylene Oxide)/Chitosan Polymer Matrix
}

\author{
R. Porras ${ }^{\mathrm{a}}$, D.V. Bavykin ${ }^{\mathrm{a}, 1}$, J. Zekonyte ${ }^{\mathrm{b}, 2}$, F.C. Walsh ${ }^{\mathrm{a}}$, R.J. Wood ${ }^{\mathrm{b}}$ \\ ${ }^{a}$ Energy Technology Research Group, Engineering Sciences, Faculty of Engineering and the \\ Environment, University of Southampton, $U K$ \\ ${ }^{b}$ National Centre for Advanced Tribology, Engineering Sciences, Faculty of Engineering and \\ the Environment, University of Southampton, UK
}

\begin{abstract}
Soft polyethylene oxide/chitosan mixtures, reinforced with hard titanate nanotubes (TiNT) by co-precipitation from aqueous solution, have been used to produce compact coatings by the "drop-cast" method, using water soluble polyethylene oxide (PEO) polymer and stable, aqueous colloidal solutions of TiNT. The effects of the nanotube concentration and their length on the hardness and modulus of the prepared composite have been studied using nanoindentation and nanoscratch techniques. The uniformity of TiNT dispersion within the polymer matrix has been studied using transmission electron microscopy (TEM). A remarkable increase in hardness and reduced Young's modulus of the composites, compared to pure polymer blends, has been observed at a TiNT concentration of $25 \mathrm{wt} \%$. The short (up to 30 minutes) ultrasound treatment of aqueous solutions containing polymers and a colloidal TiNT mixture prior to drop casting has resulted in some improvements in both hardness and reduced Young's modulus of dry composite films, probably due to a better dispersion of ceramic nanotubes within the matrix. However, further (more than 1 hour) treatment of the mixture with ultrasound resulted in a deterioration of the mechanical properties of the composite accompanied by a shortening of the nanotubes, as observed by the TEM.
\end{abstract}

Keywords: composite coating, drop-cast, nanotubes, PEO, titanium oxide,

\footnotetext{
${ }^{1}$ To whom correspondence should be addressed. Tel: + 44 2380598358, e-mail: D.Bavykin@soton.ac.uk

${ }^{2}$ Present address: School of Engineering, University of Portsmouth, UK
} 


\section{Introduction}

The reinforcement of soft ductile polymers with hard brittle ceramic materials in order to improve the mechanical properties of such composites has been explored since the dawn of polymer technology [1]. Recent advances in the synthesis of nanosized materials have further extended the range of suitable fillers for polymer matrices, with particular attention focused on fibrous nanomaterials, such as nanotubes [2] and nanofibers [3]. Carbon based elongated nanomaterials are the most popular additive to polymers $[4,5]$, as well as the promising monolayered nanosheet-like counterpart graphene $[6,7]$. The manufacture of composite materials consisting of elongated metal oxides dispersed in various matrices has also received great interest due to a wider availability of these materials and potentially better mechanical (including hardness and wear resistance) properties of such composites.

One of the promising elongated nanostructured metal oxides is titanate nanotubes (TiNT), which were first reported in 1997 by Kasuga et al. [8]. Since then, much of the research has been carried out in order to improve their synthesis and characterization [9, 10]. Such TiNT can be prepared by an alkaline hydrothermal process leading to nanotubes with a single crystal structure, small inner diameter in the range of 3-10 $\mathrm{nm}$ and a typical length exceeding hundreds of nanometres. They attract much attention due to their extraordinary properties and widespread applications in different areas such as energy conversion and storage for dyesensitized solar cells [11], lithium storage [12], fuel cells [13], hydrogen storage [14]; catalysis for isomerisation of organic compounds [15], improved capacitance [16], photocatalysis [17]; magnetic materials [18]; drug delivery [19] and composites [20]. Although initially TiNT have received less attention compared to carbon nanotubes, their development is steadily growing, due to improvements in the synthetic methods and the ease of availability of starting materials $[11,21]$.

Reinforcement of polymer matrices with nanostructured titanates such as nanotubes and nanofibres has already demonstrated an enhancement of the mechanical and tribological properties of such composites $[10,22]$. Also, such composites can demonstrate unusual optomechanical properties [23] suitable for possible energy conversion applications. Recently, the 
possibility of electrospinning a composite fibre of carbon nanotubes, chitosan and TiNT has been demonstrated [24]. Generally, there are three approaches for incorporation of solid inorganic nanostructures into a polymer matrix, namely $(i)$ mixing of dissolved polymer with colloidal solution of nanostructures followed by removal of the solvent [25], (ii) dispersion of solid nanostructures in the melt polymer [26], or (iii) in-situ synthesis of polymer from monomers in the presence of nanostructured materials [27, 28]. In addition, specialised electrochemical techniques have been developed [29]. Each method has its own advantages and limitations. For example, direct synthesis of polymer in the presence of dispersed TiNT can potentially result in a better distribution of nanostructures within the polymer matrix due to less steric restriction, allowing the monomers to compactly adsorb on the nanotube surface before polymerisation. However, such an approach is less versatile since it narrows the possible range of suitable monomers and solvents. Additionally, the added nanostructures can inhibit polymerisation.

In this work, the "drop-cast" method has been explored, using water soluble polyethylene oxide (PEO) polymer and stable aqueous colloidal solutions of TiNT. PEO consists of a repetitive structure of $\left(\mathrm{CH}_{2}-\mathrm{CH}_{2}-\mathrm{O}\right)$ and therefore poorly interacts with the negatively charged surface of TiNT, which is developed due the dissociation or polytitanic acid $\left(\mathrm{H}_{2} \mathrm{Ti}_{3} \mathrm{O}_{7}\right)$. In order to improve the dispersion of nanotubes within the matrix, additional components which are characterised by good affinity towards nanotubes (e.g. cationic polymers) can be used. Chitosan (CS) is the deacetylated derivative of chitin, which is a natural polysaccharide synthesised by a vast number of natural organisms such as crabs, shrimps, fungi and yeast $[30,31]$. When the degree of deacetylation reaches about $50 \%$, the polymer is called chitosan, which is soluble in aqueous media at $\mathrm{pH}<6$. In aqueous solutions of Chitosan, its amine groups at the $\mathrm{C} 2$ position of the D-glucosamine repeat unit can be protonated facilitating interaction of the polymer with negatively charged $-\mathrm{O}^{-}$surface groups of titanate nanotubes.

Amongst numerous reports about manufacturing of polymer composites with hard nanostructured materials, few are focused on detailed studies of distribution of these nanostructures within the matrix, which may strongly influence the mechanical performance and quality of such composites [32]. Therefore, in present work, special attention has been 
paid to careful analysis of distribution of TiNT in the final composite by methods of electron microscopy and modification of the procedure to avoid formation of large agglomerates of nanotubes within the polymer matrix.

Due to their large specific surface area, nanotubes are affected by increased van der Waals forces which can cause their agglomeration into large bundles or entangled aggregates during their synthesis [21]. The disintegration of these initial agglomerates and release of independent and separated nanotubes without significant breaking of the individual nanotubes can be achieved by long-term stirring of an aqueous suspension, resulting in the formation of a stable colloidal solution of nanotubes with a relatively high aspect ratio. The average length of the nanotubes can be further altered by using ultrasound treatment of the colloidal suspension of TiNT resulting in their shortening [12]. The mechanism of such breaking of nanotubes under ultrasound is still unclear and probably associated with the effect of cavitation [33]. The effect of the solvent density on the rate of the nanotubes shortening was studied in the present work for the first time. Ultrasonic treatment of mixed viscous solutions of polymers and nanotubes prior to solvent-casting can be an additional method to improve dispersion of nanotubes within polymers. However, identification of the optimal time is required since long term treatments of nanotubes can decrease their length and negatively affect the mechanical properties of the composite.

In the present work, we report systematic studies of mechanical properties of TiNT/PEO-CS composite films as a function of the nanotube concentration and the length. The method of nanoindentation was used for drop-cast films in order to fully characterise mechanical properties at the nanoscale. The tribological behaviour was studied using nanoscratch testing. The relationship between the indentation response and the nanoscratching tests at the nanoscale has also been investigated. Although PEO-based composites are less practical compared to commercial polymer mixtures, the established method of dispersion of titanate nanotubes within soluble polymers allows us to obtain composites with a large range of composition. The observed improvements of mechanical and tribological properties of polymers such as hardness, Young's modulus and friction coefficient suggest the great potential of nanotubular titanates as reinforcements for polymer composites. 


\section{Experimental details}

\section{Preparation of composites}

The alkaline hydrothermal method was used to synthesise TiNT based on the procedure described elsewhere [34]. 25 grams of raw titanium dioxide $\left(\mathrm{TiO}_{2}\right)$ was added to an alkaline aqueous solution of sodium hydroxide $(\mathrm{NaOH})$ and potassium hydroxide $(\mathrm{KOH}) 10 \mathrm{~mol} \mathrm{\textrm {dm } ^ { - 3 }}$ (with respect to hydroxide ions) with a 50:1 ratio of $\mathrm{NaOH}: \mathrm{KOH}$. The reaction was carried out in a perfluoroalkoxy polymer (PFA) round bottom flask at atmospheric pressure and $100^{\circ} \mathrm{C}$ for 4 days. The nanotubes were washed with an aqueous solution of $0.1 \mathrm{~mol} \mathrm{dm}{ }^{-3}$ hydrochloric acid $(\mathrm{HCl})$ until the $\mathrm{pH}$ was 2 . Distilled water was used to return to a $\mathrm{pH}$ of 5 6. After washing, TiNTs were filtered and dried under vacuum at room temperature $\left(22{ }^{\circ} \mathrm{C}\right)$ overnight.

In order to make stable colloidal suspensions of TiNT, $400 \mathrm{mg}$ of powdered TiNT was mixed with $200 \mathrm{~cm}^{3}$ of distilled water and allowed to stir at ambient temperature for 2 weeks using a magnetic stirrer with stirring rate $700 \mathrm{rpm}$. Then the suspension was allowed to settle for one day before the supernatant liquid was decanted while the precipitate at the bottom was discarded. SEM data confirm a predominantly isolated occurrence of nanotubes in the colloidal solution. The concentration of TiNT (with respect to Ti(IV)) was determined using a NEONYS-2000 UV-visible spectrophotometer (SCINCO ${ }^{\circledR}$ ) by measuring absorbance at 280 $\mathrm{nm}$ using the molar extinction coefficient, $\varepsilon=5900 \mathrm{dm}^{3} \mathrm{~mol}^{-1} \mathrm{~cm}^{-1}$ for TiNT at this wavelength. The resulting aqueous colloidal suspensions of TiNT were stable for over a year at room temperature $\left(22^{\circ} \mathrm{C}\right)$ and the concentration of TiNT typically varied between 300 and $700 \mathrm{mg} \mathrm{dm}^{-3}$.

The TiNT polymer composite was prepared by mixing aqueous solutions of nanotubes with polymer blends followed by slow evaporation of the solvent. $300 \mathrm{mg}$ of poly(ethylene oxide) (Aldrich, $\mathrm{Mw}=400 \mathrm{k}$ ) with addition of $50 \mathrm{mg}$ chitosan (Aldrich, medium Mw, 75-85\% degree of deacetylation) was dissolved in $25 \mathrm{~cm}^{3}$ of acetic acid at $2 \mathrm{wt} \%$ at room temperature $\left(22^{\circ} \mathrm{C}\right) \cdot y \mathrm{~cm}^{3}$ of stable aqueous colloidal solution of TiNT (where $y$ varied in the range from 
50 to $500 \mathrm{~cm}^{3}$ ) was added to the polymer solution under stirring. The solvent was then slowly evaporated in a rotary vacuum evaporator under reduced pressure (ca. $100 \mathrm{mbar}$ ), in a moderate temperature bath $\left(55^{\circ} \mathrm{C}\right)$, and constant spinning (120 RPM) until the final mass of the viscous solution was ca. $5 \mathrm{~g}$, which corresponds to the $6 \mathrm{wt} \%$ of PEO in the solution. The samples were cast into moulds on the flat glass slides and dried at room temperature $\left(22^{\circ} \mathrm{C}\right)$ and atmospheric pressure. The thickness of the films, which was measured with the optical 3D microscope Alicona ${ }^{\circledR}$, varied in a range from 20 to $180 \mu \mathrm{m}$. The mass ratio between nanotubes and polymer was calculated using the quantity of added TiNT and typically varied from 0 to $30 \mathrm{wt} \%$ in respect to solid polymer. The dried films were peeled off, turned upside down and fixed to a glass slide with epoxy resin, after which they were used for further studies on mechanical and tribological properties.

\section{Adjusting the length of the nanotubes}

The length of the nanotubes was controlled by breaking initially long nanotubes using ultrasound treatment of colloidal suspensions of TiNT [9]. The solutions of TiNT with or without polymer were sonicated for $0.25,0.5,1,2$ and 3 hours, in an ice bath using a HD3200 Sonopuls Ultrasonic Homogenizer (purchased from Monmouth Scientific Ltd, UK) with a 6 $\mathrm{mm}$ diameter titanium alloy probe KE76. Sonication prior casting was performed by $0.005 \mathrm{~s}$ pulses, at a frequency of $20 \mathrm{kHz}$ and an energy output of $100 \mathrm{~W}$. In order to avoid cross contamination of the sample with debris from the eroded probe tip during ultrasound treatments, the tip and the liquid samples were separated by a glass tube filled with $30 \mathrm{~cm}^{3}$ of distilled water.

In order to study the effect of solvent density on the breaking rate of TiNT, $25 \mathrm{~cm}^{3}$ of TiNT suspended in various solvents such as ethanol, water, dichloromethane, chloroform and bromoform were consecutively treated with ultrasound for 0.5, 1, 2 and 3 hours (total time), applying $0.005 \mathrm{~s}$ pulses, and using $50 \%$ of the nominal energy of the sonicator. The probe was immersed to a $1.2 \mathrm{~cm}$ depth in the liquid suspension. In order to avoid overheating of the samples, they were treated sequentially every 3 minutes and kept for another 3 minutes in an ice-bath. 


\section{Testing mechanical properties of the composites}

Mechanical properties of the composite films were tested using a Nanotest Platform $3 \mathbb{R}$ nanoindenter (Micro Materials Ltd, UK) described elsewhere [35]. A three-sided pyramidal Berkovich diamond indenter was used to measure hardness and elastic modulus of the films. The contact depth was less than $10 \%$ of the total film thickness to ensure a negligible influence of the substrate on the final results. Typical experimental conditions for indentations were: a maximum load of $1.0 \mathrm{mN}$ at a loading rate $=$ unloading rate $=20 \mathrm{mN} \mathrm{s}^{-1}$ with $30 \mathrm{~s}$ dwell at the maximum load. A matrix of 14 x 14 (196) load-controlled indentations was set in order to make representative measurements of the sample. The load-displacement curves were analysed using Oliver-Pharr method [36] with analytical software provided by Micro Materials Ltd.

The same equipment was used for nanoscratch testing. Scratching resistance was investigated by repeating alternate off-load and on-load scratch tests. A $10 \mu \mathrm{m}$ radius spherical diamond tip was used in the tests. A low load of $0.1 \mathrm{mN}$ was applied in the initial topographic scan for $500 \mu \mathrm{m}$ at a speed of $16.67 \mu \mathrm{m} \mathrm{s}^{-1}$, after which the tip was moved back to the starting position. The scratch tests were carried out along the same track applying a ramped load within the first $90 \mu \mathrm{m}$ of the path followed by a constant load of 10,20 and $50 \mathrm{mN}$ throughout the next $410 \mu \mathrm{m}$ of the track. After the on-load part of the experiments, a final topographic scan was carried out from the starting position, again at $0.1 \mathrm{mN}$ applied load. In the first and third scans, the load applied was sufficiently low that wear does not occur. Data were corrected during analysis according to the initial topographic scan. The measurements were spaced $100 \mu \mathrm{m}$ apart and repeated twice to verify homogeneity of the films. Friction coefficient was calculated from on-load experiments whereas the final topographic scan provides the residual depth data.

Thin composite films were prepared by spin coating in order to investigate the distribution of TiNT within the polymer matrix. An aliquot of $10 \mu \mathrm{L}$ of diluted TiNT/polymer solution was dropped onto carbon coated perforated copper grids attached to double sided carbon tape and spun for $60 \mathrm{~s}$ at $3000 \mathrm{rpm}$. A JEOL 3010® transmission electron microscope (TEM) operating at $300 \mathrm{kV}$ was used to examine the dispersion of nanotubes within the composite films. 


\section{Results and discussion}

\section{Scission of TiNT under ultrasonic treatments in various solvents}

Efficient dispersion of solid nanostructures into the polymer matrix using a solution route usually requires several criteria to be met, namely (a) good interfacial interactions between polymer molecules and nanofillers, and (b) availability of independent nanostructures in the solution during mixing. As synthesised, titanate nanotubes are usually agglomerated into large bundles of randomly oriented nanotubes. In order to disassemble such several micron sized agglomerates and disperse single nanotubes into the colloidal solution, ultrasound treatment of TiNT powder suspended in the suitable solvents is widely used. However, such a treatment was found to result not only in disintegration of nanotube bundles but also in the breaking of each individual nanotube, leading to the reduction of their average length [9]. The average length of nanotubes added to composite materials can affect the mechanical properties of the final product. Therefore, in order to minimize nanotube shortening, the effect of operational conditions during ultrasound treatment of suspended TiNT on their breaking rate was studied. In particular, based on our previous observations that the rate of the breaking is different in different solvents, the effect of the solvent density on the breaking rate of nanotubes was systematically studied with a wide range of densities from $0.798 \mathrm{~g} \mathrm{~cm}^{-3}$ (ethanol) to $2.89 \mathrm{~g} \mathrm{~cm}^{-3}$ (bromoform).

Stable colloidal suspensions of long nanotubes were prepared by applying minimal mechanical impact on the nanotubes using several days stirring of TiNT suspended in various solvents. It was found that the kinetic curve of nanotubes concentration in the solution as a function of the stirring time has a sigma shape with a short several days induction time after which a rapid growth in concentration is observed followed by a plateau (see Supporting Information Figure S1). All samples of long colloidal titanate nanotubes were obtained by 2 week stirring in various solvents. Unlike in ethanol and water where neat nanotubes were used, the dispersion of nanotubes in dichloromethane, chloroform and bromoform required a prior functionalization of nanotubes with CTAB. This was achieved by adsorption of dissolved CTAB from its aqueous solution $\left(0.1 \mathrm{~mol} \mathrm{dm}^{-3}\right)$ on the surface of the nanotubes at 
room temperature for 24 hours followed by washing the nanotubes with excess distilled water and drying.

Once the nanotubes were well dispersed, ultrasound was applied to the samples. Figure 1 shows SEM images of titanate nanotubes deposited on the surface of silicon wafer from the colloidal solutions before and after their treatment with ultrasound for 3 hours. SEM data confirm that most of the nanotubes in the colloidal solution are not in bundles but isolated. Only a small number of TiNT agglomerates can be found in the samples. Original nanotubes before treatment with ultrasound are characterized by a relatively large length, which decreases after ultrasound treatment, as shown in Figure 1b. Surprisingly, the same ultrasound treatment of colloidal nanotubes in ethanol solution results in almost no change in average length of nanotubes, as seen in Figure 1c.

Figure 2 shows the length distributions of titanate nanotubes in various solvents before and after their treatment with ultrasound for 3 hours. These histograms were built by careful analysis of SEM images of titanate nanotubes deposited on the flat support (silicon wafer) with a relatively low surface density of nanotubes (in order to avoid overlapping of nanotubes and their aggregation). The length of more than 100 individual nanotubes was digitally measured in order to make a statistically significant representation of each experimental point. All histograms are characterised by an asymmetrical shape with relatively wide width and long tail at large length.

When ethanol was used as a solvent for TiNT, its ultrasound treatment has resulted in almost no change in length distribution histograms (see Figure 2a). In contrast, the same ultrasonic treatment of aqueous suspension of TiNT has shifted the length distribution towards lower values (see Figure 2b) indicating efficient breaking of nanotubes in water. In denser solvents, such as dichloromethane and chloroform (Figure $2 \mathrm{c}$ and Figure $2 \mathrm{~d}$ respectively), the effect of ultrasound is even more pronounced. For example, relatively long nanotubes (up to $250 \mathrm{~nm}$ ) were observed after 3 hours treatment in water, whereas, only a few nanotubes longer than $150 \mathrm{~nm}$ could be detected quantitatively in dichloromethane and practically no nanotubes longer than $120 \mathrm{~nm}$ in chloroform after 3 hours of ultrasound treatment. 
Figure 3 shows the average length reduction of titanate nanotubes during ultrasonic treatment of colloidal TiNT in various solvents such as ethanol, water, dichloromethane and chloroform. All kinetic curves follow a non-exponential decay curve which stabilises at a certain length. Initial average length of nanotubes rapidly decreases and after 2 hours of ultrasonic treatment there is no significant change in nanotubes length. This final length varies in different solvents and the observed tendency suggests that the denser the solvent, the shorter the terminal length of nanotubes. However, as indicated by the inset graph in Figure3, for the two solvents with the highest density (chloroform and bromoform) the difference in terminal length is negligible, despite the difference in their densities.

Although the phenomenon of nanotube scission under ultrasound treatment has been widely reported [37, 38], the exact mechanism of their fracture is not well understood. Generally it is considered that the viscous drag forces induced by bubble collapse during cavitation are balanced by mechanical tensile stresses, which are maximal near the centre of the nanotubes. Nanotubes can be broken when the stress exceeds the nanotube tensile strength and the average length of the nanotubes $L(t)$ in this case will be proportional to the time of sonication $t^{-n}$ where $n \cong 0.2$ [39]. We have found, however, that kinetic curves of titanate nanotubes scission under sonication (see Figure 3) data do not fit such time power dependence probably due to the fact that it was derived for the nanotubes axially oriented towards the centre of the collapsing bubble. Recently, Pagani et al [40] has reported that if nanotubes are oriented parallel to the surface of the collapsing bubble, the rate of the nanotube buckling is different and therefore $L(t)$ is proportional to $t^{-n}$ where $n \cong 0.5$. Usually, long nanotubes have a buckling mechanism of scission whereas short nanotubes more likely to orient axially and break as they stretch. As a result the overall kinetic curve of $L(t)$ decay has a rather steep initial part followed by the long tail where the rate of nanotube breakage is reduced. The apparent kinetic curve of titanate nanotube scission, characterised by an initial rapid decrease of average length, probably demonstrates a switch from one mechanism of nanotube scission to another. 
The initial rate of nanotube shortening in various solvents under ultrasound is also correlated with the density of the media. Apparent initial breaking rate (in nm per hour) as a function of solvent density is shown in the Figure 3 inset. For the ethanol, water, dichloromethane and chloroform the rate of nanotube breaking grows almost linearly with the density of the solvent. However, after chloroform, a further increase in density has no effect on the breaking rate. Such an effect of solvent density on the rate of nanotube scission has not been systematically investigated hitherto. There are several possible reasons for acceleration of nanotube scission in denser solvents. Firstly, the replacement of the solvent may change the maximal size of the bubbles resulting in a different level of forces occurring during their collapse [38]. Such alteration of the amount of acoustic energy absorbed by the solvent would eventually result in a variation of thermal energy added to the solution. Table 1 shows temperature rise $(\Delta T)$ during sonication of $25 \mathrm{~cm}^{3}$ of all solvents for 1 minute in adiabatic conditions. The initial temperature of the solvents was $22{ }^{\circ} \mathrm{C}$ to avoid an error due to evaporation. The amount of acoustic energy converted to thermal energy can be estimated as $\Delta Q=\rho V \mathrm{C}_{\mathrm{p}} \Delta T$ where $\rho$ is density, $V$ is volume and $\mathrm{C}_{\mathrm{p}}$ is specific heat of the solvents (see Table 1). It was found that the heat added during sonication of solvents varies between 0.35 and $0.76 \mathrm{~kJ}$ without correlation with density of the solvent. The amount of energy is relatively low to initiate the crystal phase transformation, which can be observed under ball milling [41]. Insignificant difference between solvents probably suggests that the regime of cavitation and the size of the bubbles are almost the same in all selected media.

A second possible difference between solvents could be associated with the strength of the interaction between the surface of the nanotubes and the liquids affecting the values of the friction forces. In general, the value of drag force can be strongly affected by the type of the flow in the media. It has been shown that at this range of nanotube sizes, a switch between laminar to turbulent flow is possible $[39,42]$. Table 1 shows the density $(\rho)$ and viscosity $(\mu)$ of the solvents at room temperature $\left(22^{\circ} \mathrm{C}\right)$. The ratio of the density over the viscosity $(\rho / \mu)$ grows from 0.73 to 2.73 . Assuming that the size of the bubbles is the same for all solvents, the Reynolds number is increasing almost 4 fold from ethanol to chloroform. Such an increase not only increases the level of stress affecting the nanotubes during collapse of the cavitation bubble but also can potentially transform laminar flow around the nanotubes to turbulent. 


\section{Distribution of TiNT within polymer matrix}

Since we established a method of dispersion of TiNT in several solvents producing stable colloidal suspensions of principally isolated nanotubes, attempts at mixing such suspensions with polymer solutions were made. It was found that aqueous suspensions of nanotubes can be mixed at room temperature with aqueous acetic solutions of PEO and CS into homogeneous mixtures without precipitation of nanotubes. Due to a limited TiNT concentration in the suspension, a relatively large volume has to be added to the polymer solution in order to incorporate a high proportion of ceramic nanostructures, which in turn dilutes the mixture. However, this mixture can be concentrated by slow evaporation of the water at reduced pressure, also without coagulation of nanotubes, until a required concentration and viscosity is achieved prior to casting.

In order to determine the degree of uniformity of TiNT dispersed within the PEO/chitosan polymer matrix, a composite thin film has been deposited directly onto the copper grid (TEM specimen) by spin coating from a diluted solution. Provided that the thickness of the film is small enough for the electron beam in the TEM to penetrate it, one can obtain the cross section of the composite film, clearly identifying contrast dark titanate nanotubes embedded into the polymer matrix. Data show that nanotubes are randomly oriented and most of them are isolated as single tubes whereas some others are assembled into small aggregates, the percentage of which is insignificant. Figure 4a shows a representative TEM image of a thick composite film containing $12.5 \mathrm{wt} \%$ TiNT uniformly dispersed within the mixture of polymers (PEO:CS=6:1). The thickness of the film is relatively high, which results in superimposition of nanotubes and introduces difficulties in obtaining a good focus over all objects in the image. Nevertheless, it is clear that the local density of nanotubes within the polymer matrix is approximately the same at different parts of the sample, indicating a good distribution of nanotubes. 
Figure $4 \mathrm{~b}$ shows a TEM image of rare imperfection in the film, a crack probably induced by its mechanical tearing. Interestingly, the nanotubes are aligned in the perpendicular direction to the crack inside the stretched fibrils of the matrix. We can speculate here that improvement of the polymer's mechanical properties can be associated with the formation of such fibrils containing aligned nanotubes, which might hinder the propagation of the crack and facilitate the stability of the composite film structure.

\section{Mechanical and tribological properties of the composites}

Hardness $(\mathrm{H})$ and reduced Young's modulus (Er) of the composites has been measured using the nanoindentation technique. Both the loading and unloading curves were measured and the hardness and modulus values (calculated from the unloading part of the curve) were repeatedly measured many times for each sample. Despite this, the results show a relatively high degree of scatter. The method of nanoindentation can still be used for the investigation of polymer film uniformity at the nano-scale. Figure 5 shows the extracted values of hardness $(\mathrm{H})$ and reduced Young's modulus $(\mathrm{Er})$ as a function of concentration of TiNT in the composite. The concentration of TiNT in weight percent in solid film was calculated from the dry mass of nanotubes added to polymer mixture (PEO and CS in 6:1 mass ratio). The content of nanotubes in the dry composite was: $0,3,6,9,12.5,15,20,25$ and 30 wt $\%$.

It was found that addition of TiNT to the polymer resulted in an initially rapid increase in hardness and modulus compared to the pure polymer mixture. The composite film filled with $25 \mathrm{wt} \%$ of nanotubes was found to be approximately 2.6 times harder compared to the neat polymer blend and 3.4 times stiffer (see Table S1 in Supporting Information). It is possible that a further increase of nanotube concentration in the polymer blend may lead to particle aggregation and poor dispersion of the reinforcing phase at high concentrations of nanotubes leading to some deterioration of the mechanical properties of the composite.

It is common practice during distribution of solid particles within polymer solutions to use ultrasound to accelerate mixing and improve uniformity of the phase [43]. However, taking into account that such treatment can also reduce the average length of nanotubes [9], it is 
essential to know how alteration of the nanotubes length affects the mechanical properties of the composite. For this purpose, polymer solutions (PEO:CS 6:1) containing TiNT were stirred overnight and then treated with ultrasound for $0,0.25,0.5,1,2$ and 3 hours followed by drop-casting of the solution on the glass slides. The concentration of TiNT in dry composite was $4.7 \mathrm{wt} \%$. The measured values of hardness and Young's modulus for the obtained composite films are shown in Figure 6 and Table S2 in Supporting Information. The blank data point represents hardness and Young's modulus for neat polymer blend without addition of nanotubes. After addition of long titanate nanotubes at $4.7 \mathrm{wt} \%$ to the polymer blend, without ultrasound treatment, both hardness and Young's modulus are increased approximately 1.5 times. Short time sonication of an aqueous mixture of TiNT with polymers further improves the mechanical properties of the final composite. The highest value of hardness $(0.146 \mathrm{GPa})$ is observed in the sample treated for 30 minutes ultrasound whereas the highest value of Young's modulus $(3.97 \mathrm{GPa})$ was detected in the sample sonicated for 1 hour.

These tendencies contrast recently reported correlations with carbon nanotubes suggesting that the longer the carbon nanotube, the better the mechanical properties of their polymer composites [44]. As demonstrated in the previous section, sonication of colloidal suspensions of TiNT reduces the average length of nanotubes relatively quickly and rapid deterioration of the mechanical properties of composite under ultrasound due to the scission of nanotubes would be expected. However, the observed improvement of mechanical properties of the composite after short exposure to ultrasound suggests that the benefit of better dispersion of the reinforcing phase within the matrix surpasses the detrimental effect of nanotubes length reduction at least for the short duration of the ultrasonic treatments.

In contrast, continuation of sonication reverses the effect. Samples obtained after 3 hours of ultrasound treatment showed lower values of hardness and modulus, which is related to the reduction of the nanotubes length, as confirmed by TEM. Figure 7 shows length distribution histograms built from TEM images of composite films reinforced with TiNT at $4.7 \mathrm{wt} \%$ for samples pre-treated with ultrasound for 0 and 3 hours. The shift of the distribution after sonication indicate that despite the lower Reynolds number in the polymer solution 
(compared to pure water) nanotubes still undergoe scission under ultrasonication, although probably at a lower rate.

All prepared composite samples made of PEO:CS 6:1 with a content of TiNT from 0 to 30 wt $\%$ have been subjected to nanoscratch testing. In general, mechanical and tribological properties are correlated. For that reason it might be assumed that films with better mechanical characteristics would exhibit the best scratch resistance. Figure S2 in Supporting Information shows graphs of average residual depths measured from the topographic scan on the scratched track as a function of TiNT content under three different loads $(10,20$ and 50 $\mathrm{mN})$. Residual depth was found to be lower in films filled with TiNT at 25 and $30 \mathrm{wt} \%$, both of which exhibited the best mechanical properties in the aforementioned nanoindentation analysis. As the concentration of nanotubes is increased, the scratching track seems to be less damaged for the film containing TiNT at $30 \mathrm{wt} \%$, reflecting the fact that hardness and scratch resistance are related.

Friction coefficient in composite films made of PEO:CS 6:1 as a function of TiNT concentration (from 0 to $30 \mathrm{wt} \%$ ) under the three different loads (10, 20 and $50 \mathrm{mN}$ ) is shown in Figure 8. An increase of friction coefficient occurs at low content of nanotubes, with a decrease at high nanotube content, reaching the minimum value for the composite filled with $30 \mathrm{wt} \%$ TiNT. It is also observed that friction coefficient is always higher as the applied load is increased from 10 to $50 \mathrm{mN}$. These results are in agreement with the data of residual depth showing that the composite film with a content of TiNT at $30 \mathrm{wt} \%$ is more scratch resistant as well as exhibiting the lowest friction coefficient.

Nanoscratch testing was also carried out on a 6:1 ratio PEO:CS 4.7 wt $\%$ TiNT composite film treated with ultrasound. Figure S3 in Supporting Information shows the average residual scratch depth under 10, 20 and $50 \mathrm{mN}$ applied load as a function of sonication time. Friction coefficient was calculated from the on-load scan in the scratch track under various loads and the data are shown in Figure S4 in Supporting Information. An initial increase of friction coefficient occurs after a short ultrasound treatment, after which a steady decrease is produced until a minimum is reached for 1 hour of ultrasound treatment. Friction coefficient 
rises after a further use of ultrasound. In agreement with previous data, friction coefficient is found to be always higher when the applied load increases from 10 to $50 \mathrm{mN}$. Overall, the effect of ultrasound on the tribological properties of the composite is insignificant.

\section{Conclusions}

In this paper, titanate nanotubes synthesised by the alkaline hydrothermal method were used to reinforce $\mathrm{PEO} / \mathrm{CS}$ polymer blends at controlled loadings. It has been demonstrated by TEM images that independent nanotubes were well dispersed within the polymer matrix so effectively that they might hinder the propagation of cracks induced by mechanical tearing. We can speculate that formation of fibrils of polymer and aligned nanotubes perpendicular to the crack direction might help to maintain stability of such composite films. Polymer composite film PEO:CS 6:1 with a 25 wt\% content of TiNT was found to be approximately 2.6 times as hard as the neat polymer blend and 3.4 times stiffer. Further addition of nanotubes had a negative effect on the mechanical properties due to a poor distribution and nanotube aggregation. Composite films reinforced with TiNT at $30 \mathrm{wt} \%$ exhibited the best scratch resistance due to improved mechanical properties and led to a decrease in the friction coefficient.

A comparative study of titanate nanotubes dispersed in various solvents was carried out in order to investigate comminution of such nanotubes under ultrasound treatment as a function of solvent density and sonication time in the absence of polymer. It has been demonstrated that the scission rate of nanotubes is dependent on the density of the solvent, which is much higher at the beginning, reflecting the fact that fragmentation of nanotubes is more likely to occur in long nanotubes.

Solutions of titanate nanotubes dispersed in polymer solutions were treated with ultrasound prior to casting. Although the breaking rate is expected to vary due to different experimental conditions, it has been demonstrated that nanotubes break under ultrasound treatment. PEO:CS 6:1 solutions loaded with TiNT $4.7 \mathrm{wt} \%$ were subjected to ultrasound. A better nanotube dispersion as well as shortening of nanotube length takes place simultaneously. One 
hour of ultrasound treatment gave rise to a composite film 1.5 times harder and stiffer as well as the best scratch resistant behaviour due to a low friction coefficient.

\section{Acknowledgements}

The authors gratefully acknowledge financial support from the Defence Science and Technology Laboratory (Dstl) (grant DSTLX1000064088), Ministry of Defence of the United Kingdom. Published with the permission of the Defence Science and Technology Laboratory on behalf of the Controller of HMSO. 
Table 1. Properties of the solvents, their temperature change $(\Delta \mathrm{T})$ during treatment of $25 \mathrm{~cm}^{3}$ for 1 minute with ultrasound and estimated thermal energy delivered to the solution $(\Delta \mathrm{Q})$.

\begin{tabular}{ccccccc}
\hline Solvent & $\begin{array}{c}\text { Density, } \boldsymbol{\rho} / \mathbf{g} \\
\mathbf{c m}^{-3}\end{array}$ & $\begin{array}{c}\text { Viscosity, } \\
\boldsymbol{\mu} / \mathbf{m P a s}\end{array}$ & $\boldsymbol{\rho} / \boldsymbol{\mu}$ & $\begin{array}{c}\mathbf{C}_{\mathbf{p}} / \\
\mathbf{J ~ g}^{-1} \mathbf{K}^{-\mathbf{1}}\end{array}$ & $\boldsymbol{\Delta T / \mathbf { K }}$ & $\boldsymbol{\Delta Q / \mathbf { k J }}$ \\
\hline Ethanol & 0.789 & 1.082 & 0.729 & 2.44 & 11.9 & 0.570 \\
Water & 0.998 & 0.89 & 1.121 & 4.183 & 7.3 & 0.762 \\
Dichloromethane & 1.33 & 0.413 & 3.22 & 1.21 & 8.7 & 0.350 \\
Chloroform & 1.48 & 0.542 & 2.73 & 1.05 & 10.7 & 0.416 \\
Bromoform & 2.89 & 1.857 & 1.56 & 0.52 & N/A & N/A \\
\hline
\end{tabular}

a) Viscosity taken at $25^{\circ} \mathrm{C}$. 


\section{Figure captions}

Figure 1. SEM images of titanate nanotubes deposited on silicon wafer from colloidal solutions: (a) original nanotubes from aqueous suspension, (b) after 3 hours treatment with ultrasound in water, (c) after 3 hours ultrasonication in ethanol.

Figure 2. Length distribution histograms of titanate nanotubes before and after treatment with ultrasound for 3 hours suspended in (a) ethanol, (b) water, (c) dichloromethane, and (d) chloroform. The histograms were constructed by measuring the number of nanotubes of a particular length from SEM images.

Figure 3. The mean length of nanotube decay under ultrasonic treatment of ethanol ( $\square$ ), aqueous $(\bullet)$, dichloromethane $(\diamond)$, chloroform $(\square)$ and bromoform $(\bigcirc)$ suspensions of TiNT at room temperature $\left(22{ }^{\circ} \mathrm{C}\right)$. Inset: breaking rate $v s$. solvent density.

Figure 4. TEM images of titanate nanotubes (12.5 wt\%) dispersed in polymer matrix (PEO:CS=6:1 wt\%); (a) representative homogeneous film, (b) crack in the film with debris.

Figure 5. Hardness (left axis) and reduced Young's modulus (right axis) of composite films PEO:CS 6:1 reinforced with TiNT at the following concentration of nanotubes: $0,3,6,9$, $12.5,15,20,25,30 \mathrm{wt} \%$.

Figure 6. Hardness (left axis) and reduced Young's modulus (right axis) of dry composite films PEO:CS 6:1 reinforced with $4.7 \mathrm{wt} \%$ of TiNT 4.7. The mixed solution of polymer with nanotubes was treated with ultrasound for $0.25,0.5,1,2$ and 3 hours prior to casting.

Figure 7. Length distribution histograms of $4.7 \mathrm{wt} \%$ TiNT dispersed in PEO:CS 6:1 without ultrasound (top) and with 3 hours ultrasound treatment (bottom).

Figure 8. Friction coefficient of composite films of PEO:CS 6:1 reinforced with TiNT at various concentrations. The friction coefficient was determined from nanoscratching experiments at applied loadings $10 \mathrm{mN}(\bullet), 20 \mathrm{mN}(\bullet)$ and $50 \mathrm{mN}(\bullet)$. 

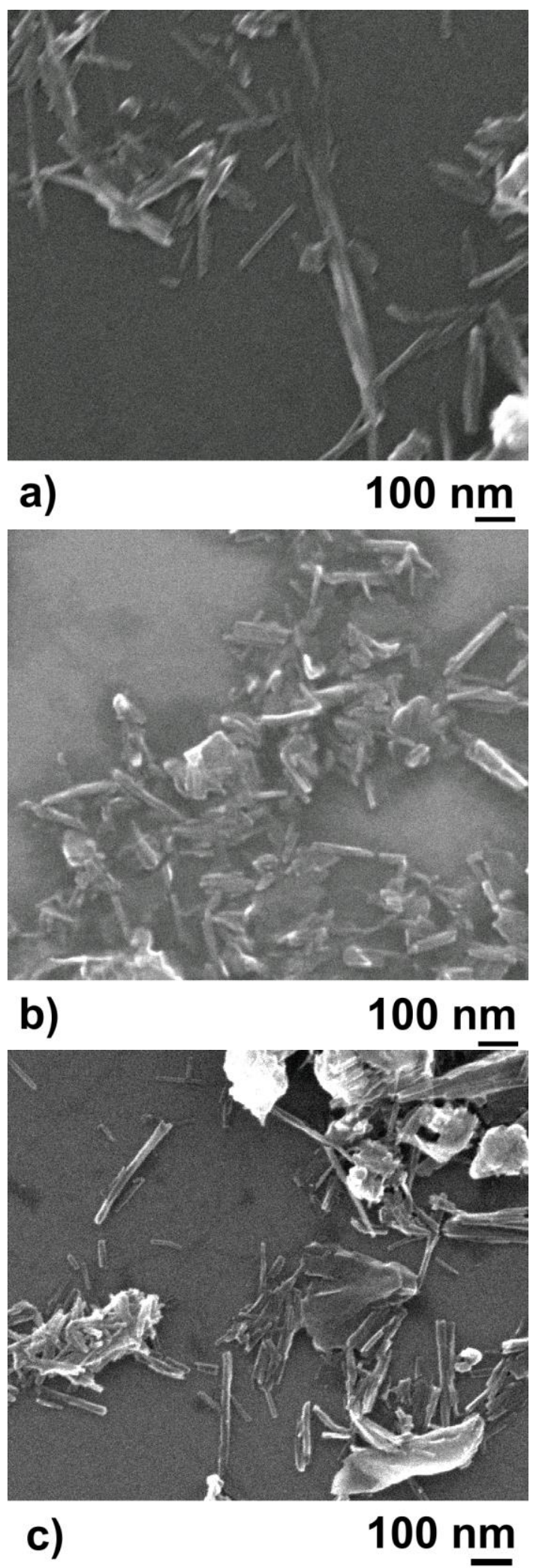

Figure 1. SEM images of titanate nanotubes deposited on silicon wafer from colloidal solutions: (a) original nanotubes from aqueous suspension, (b) after 3 hours treatment with ultrasound in water, (c) after 3 hours ultrasonication in ethanol. 

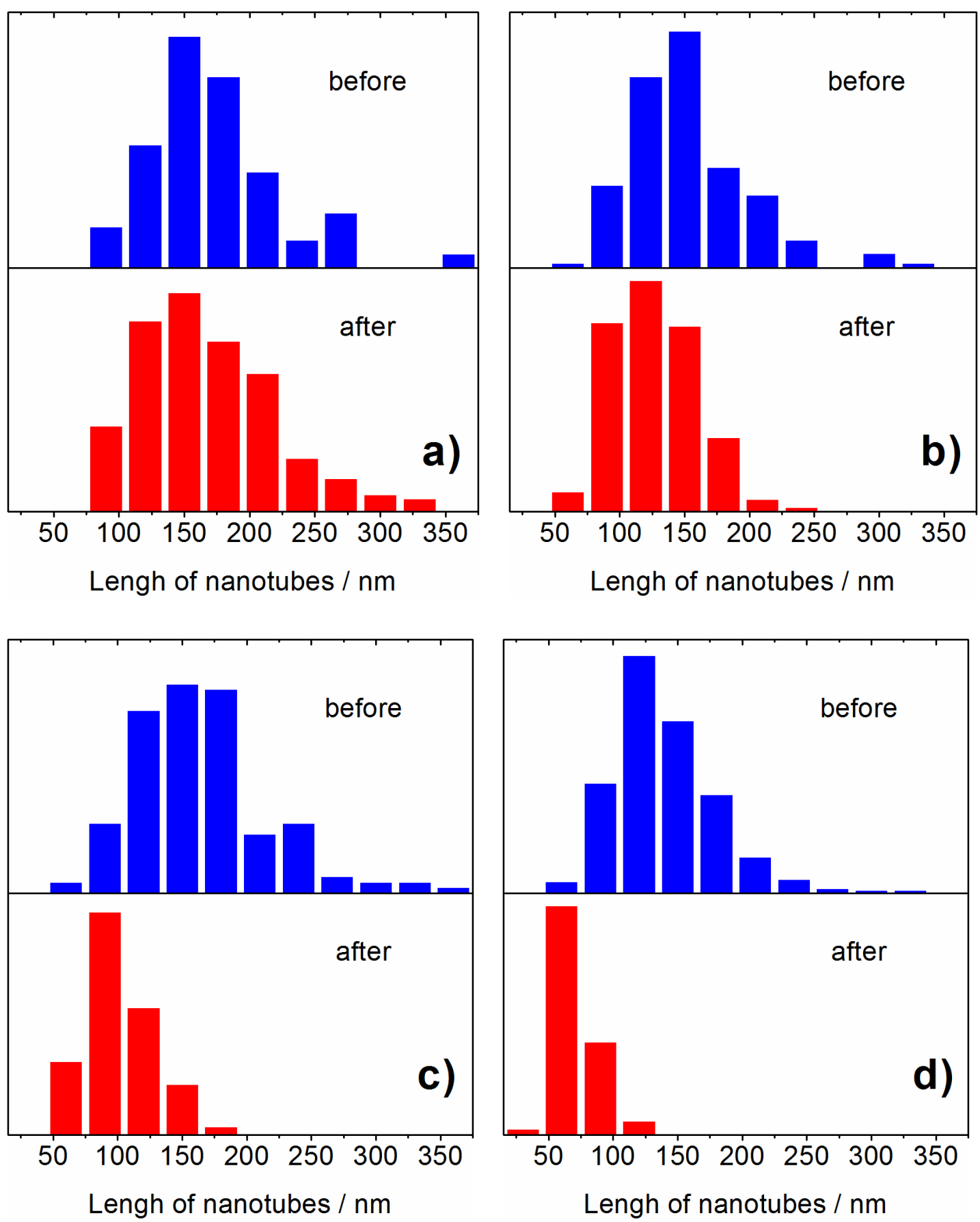

Figure 2. Length distribution histograms of titanate nanotubes before and after treatment with ultrasound for 3 hours suspended in (a) ethanol, (b) water, (c) dichloromethane, and (d) chloroform. The histograms were constructed by measuring the number of nanotubes of a particular length from SEM images. 


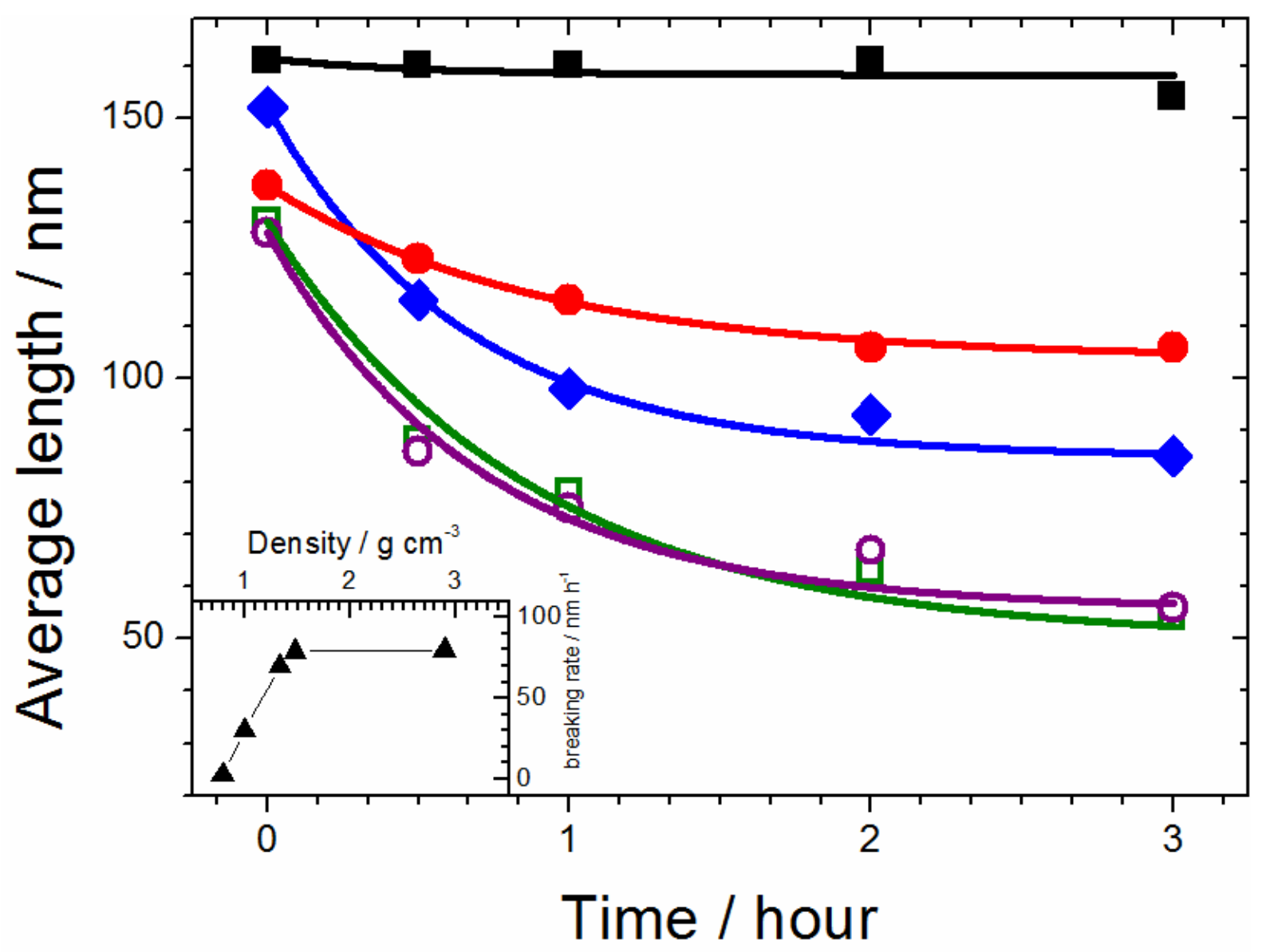

Figure 3. The mean length of nanotube decay under ultrasonic treatment of ethanol ( $\mathbf{\square})$, aqueous $(\bullet)$, dichloromethane $(\diamond)$, chloroform $(\square)$ and bromoform $(\bigcirc)$ suspensions of TiNT at room temperature. Inset: breaking rate $v s$. solvent density. 

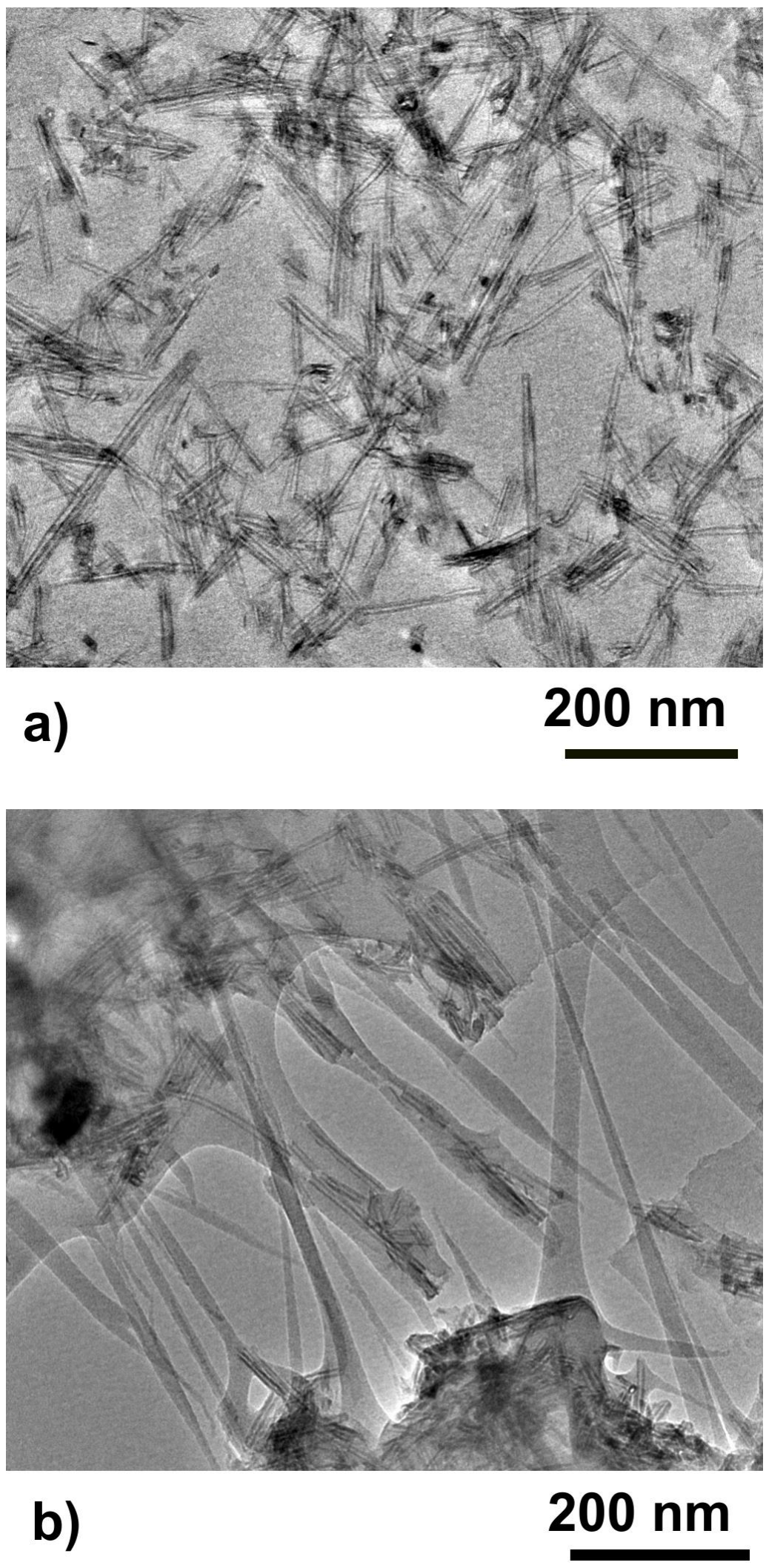

Figure 4. TEM images of titanate nanotubes (12.5 wt \%) dispersed in polymer matrix (PEO:CS=6:1 wt\%); (a) representative homogeneous film, (b) crack in the film with debris. 


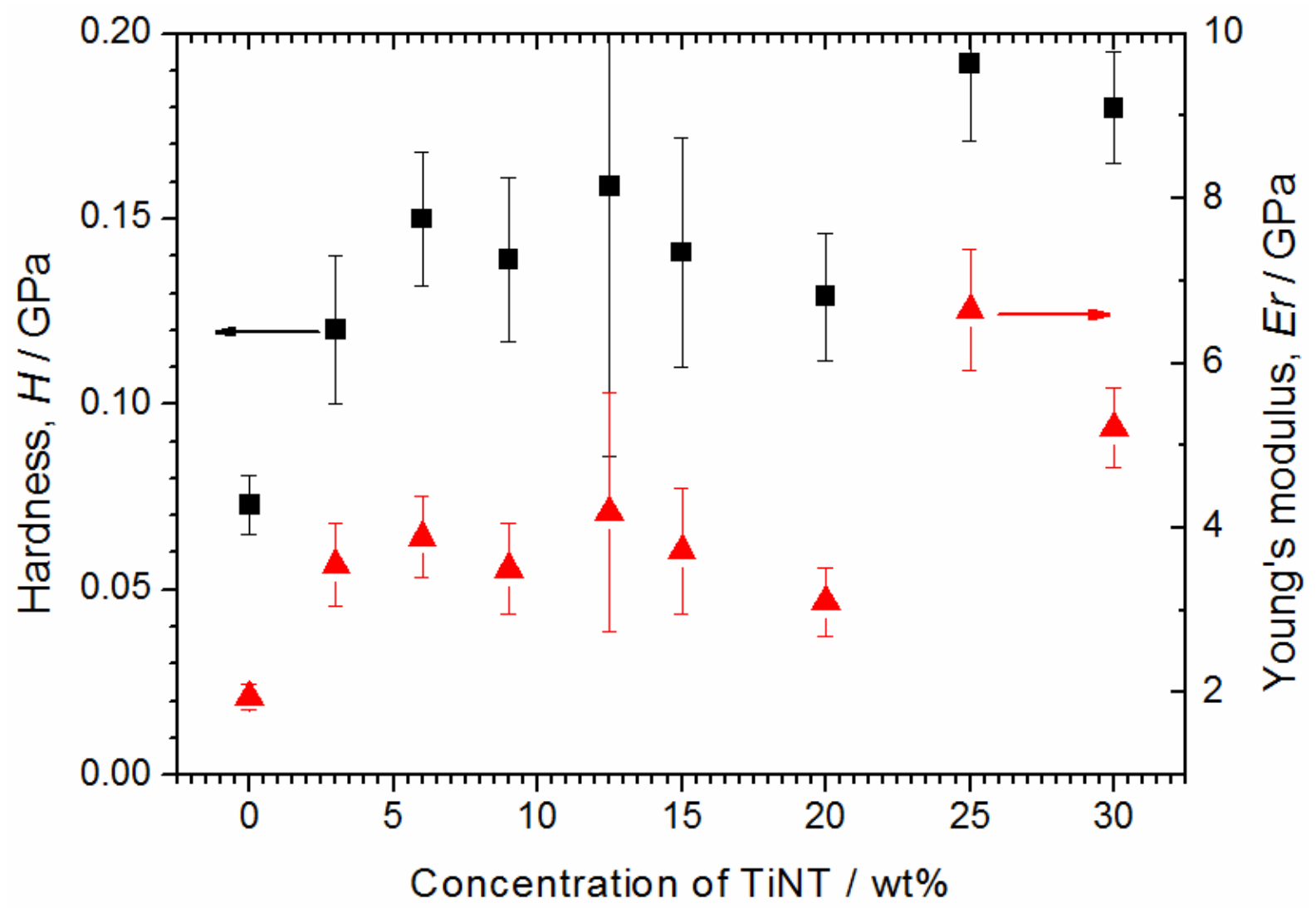

Figure 5. Hardness (left axis) and reduced Young's modulus (right axis) of composite films PEO:CS 6:1 reinforced with TiNT at the following concentration of nanotubes: 0, 3, 6, 9, $12.5,15,20,25,30 \mathrm{wt} \%$. 


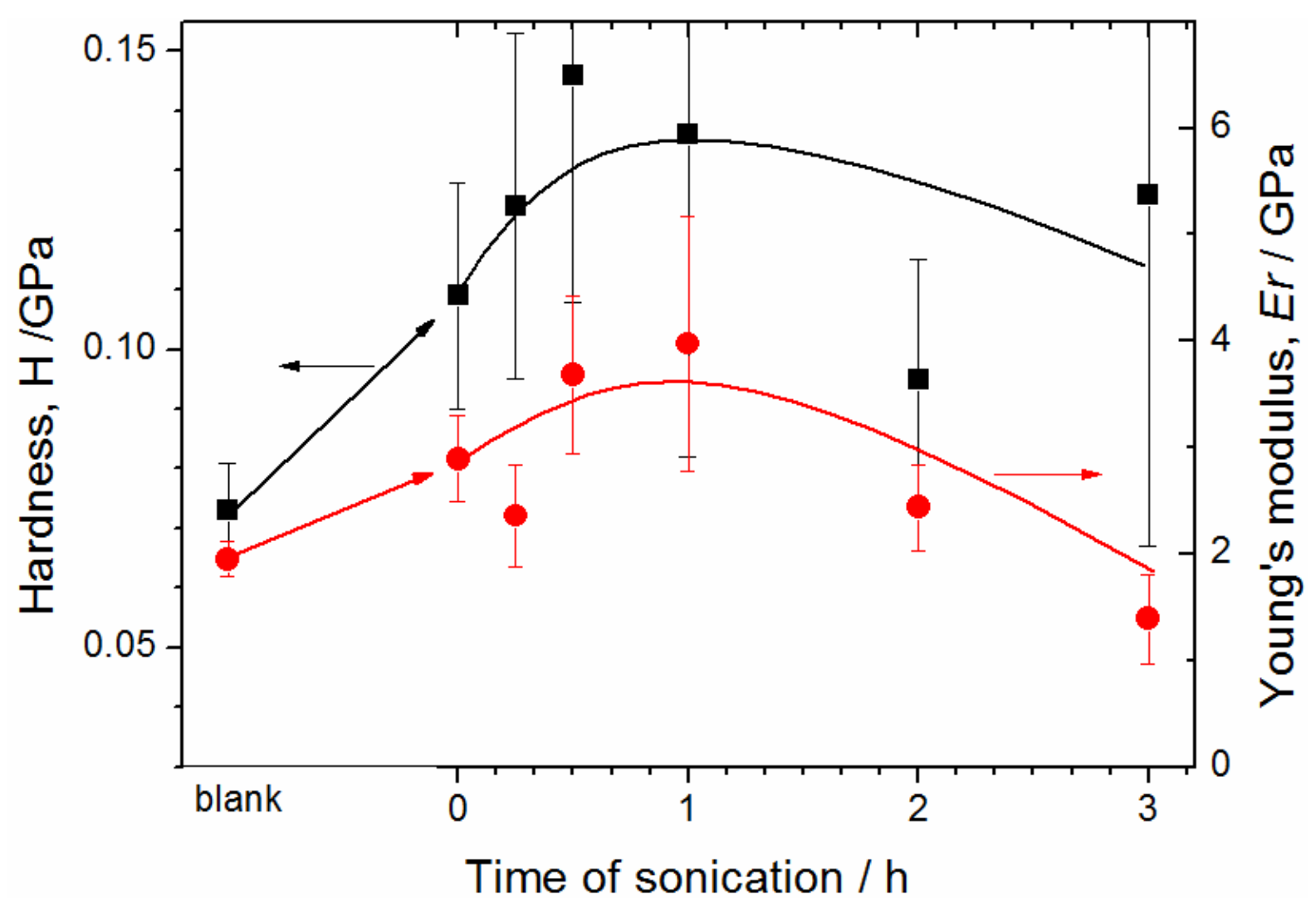

Figure 6. Hardness (left axis) and reduced Young's modulus (right axis) of dry composite films PEO:CS 6:1 reinforced with $4.7 \mathrm{wt} \%$ of TiNT 4.7. The mixed solution of polymer with nanotubes was treated with ultrasound for $0.25,0.5,1,2$ and 3 hours prior to casting. 


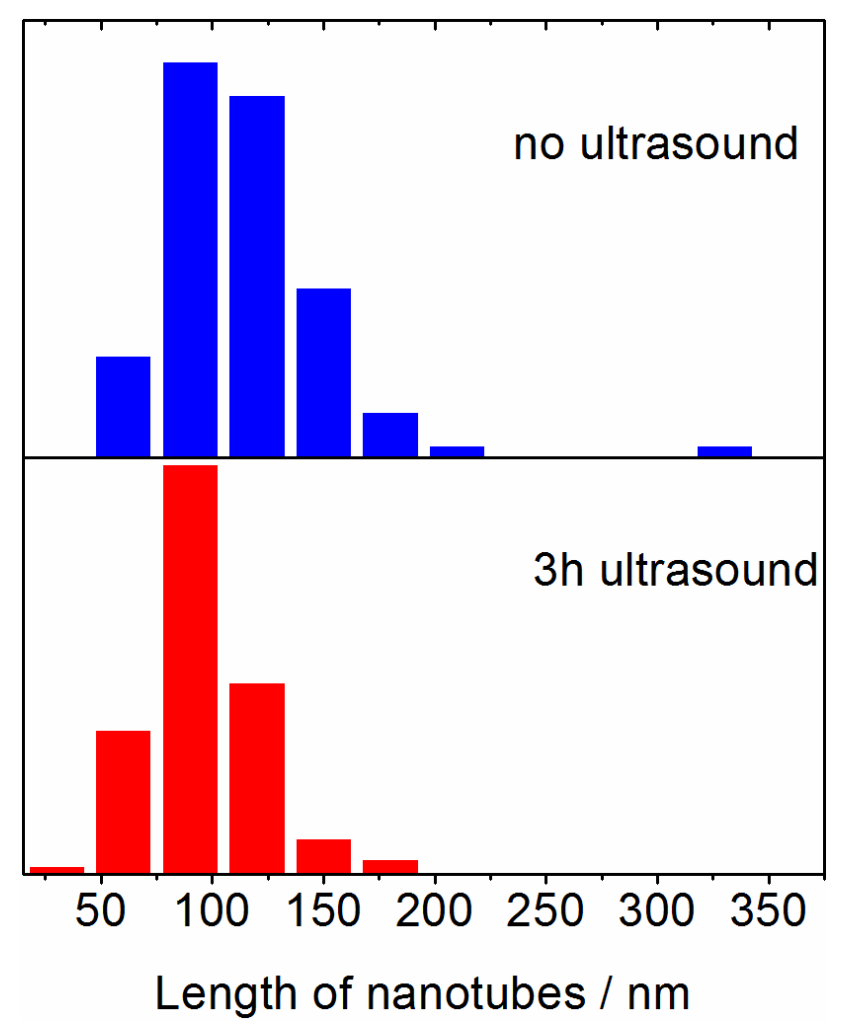

Figure 7. Length distribution histograms of $4.7 \mathrm{wt} \%$ TiNT dispersed in PEO:CS 6:1 without ultrasound (top) and with 3 hours ultrasound treatment (bottom). 3 hours. 


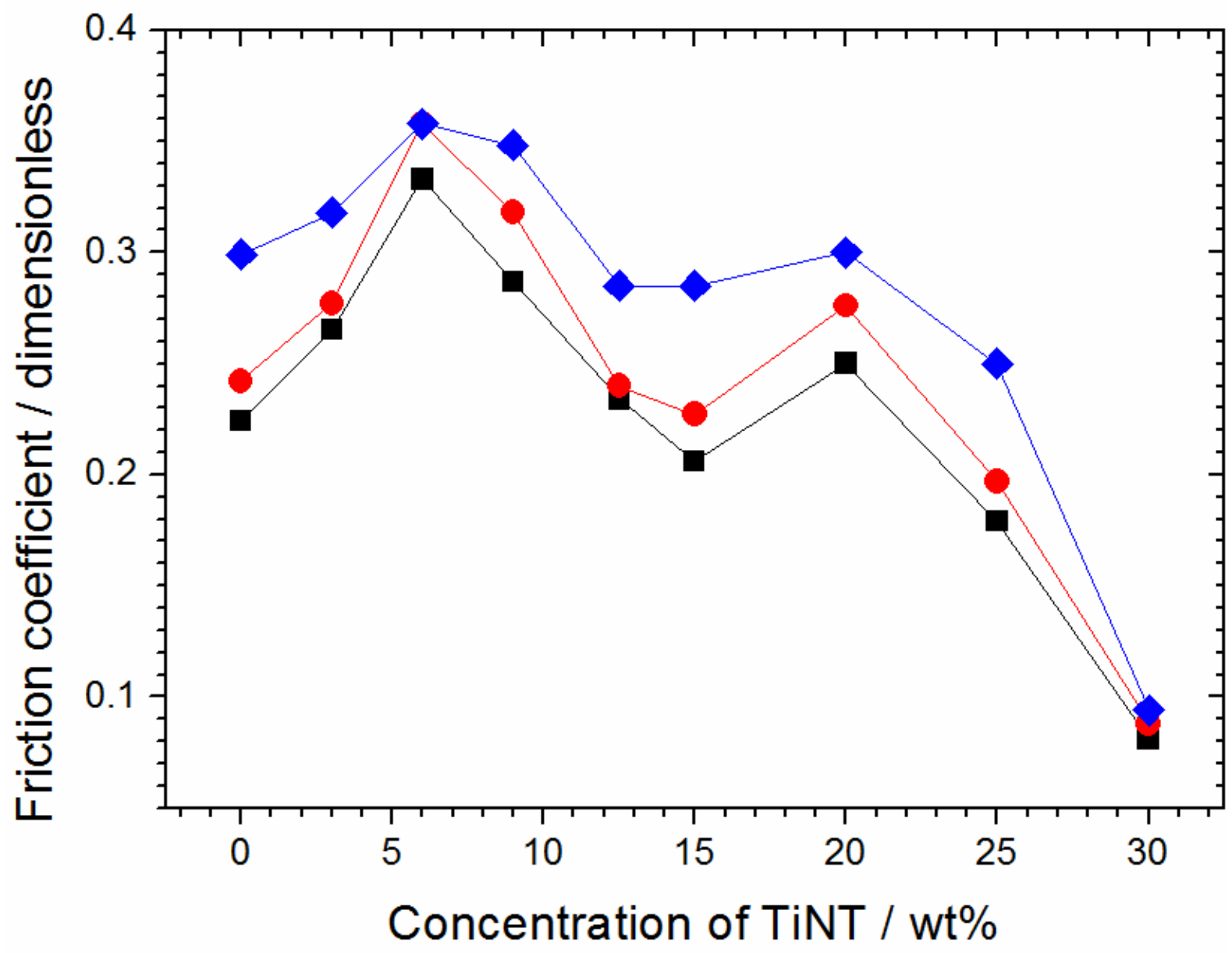

Figure 8. Friction coefficient of composite films PEO:CS 6:1 reinforced with TiNT at various concentrations. Friction coefficient was determined from nanoscratching experiments at applied loads $10 \mathrm{mN}(\boldsymbol{\square}), 20 \mathrm{mN}(\bullet)$ and $50 \mathrm{mN}(\bullet)$. 


\section{References}

1. V. Mittal. Advances in Polymer Nanocomposite Technology. Nova Science Publishers. 2010.

2. U. Khan, F.M. Blighe, J.N. Coleman. J. Phys. Chem. C. 2010. 114, 11401.

3. L.R. Xu, V. Bhamidipati, W.-H. Zhong, J. Li, C.M. Lukehart, E. Lara-Curzio, K.C. Liu, M.J. Lance. J. C. Mat. 2004. 38, 18.

4. L. Hu, D.S. Hecht, G. Gruner. Chem. Rev. 2010. 110, 10, 5790.

5. J.N. Coleman, M. Cadek, R. Blake, V. Nicolosi, K.P. Ryan, C. Belton, A. Fonseca, J.B. Nagy, Y. K. Gun'ko, W.J. Blau. Adv. Funct. Mat. 2004. 14, 8, 791.

6. U. Khan, P. May, A. O’Neill, J.N. Coleman. Carbon. 2010. 48, 14, 4035.

7. L. Gong, I.A. Kinloch, R.J. Young, I. Riaz, R. Jalil, K.S. Novoselov. Adv. Mater. 2010. 22, 2694.

8. T. Kasuga, M. Hiramatsu, A. Hoson, T. Sekino, K. Niihara. Langmuir. 1998. 14, 3160.

9. D.V. Bavykin, J.M. Friedrich, F.W. Walsh. Adv. Mater. 2006. 18, 2807.

10. D.V. Bavykin, F.W. Walsh. Titanate and Titania Nanotubes. RSC Publishing, 2010.

11. D.V. Bavykin, K.E. Redmond, B.P. Nias, A.N. Kulak, F.C. Walsh. Aust. J. Chem. 2010. 63,270 .

12. D.V. Bavykin, F.C. Walsh. J. Phys. Chem. C. 2007. 111, 40, 14644.

13. J. Geng, Z. Jiang, J. Wang, Y. Shi, D. Yang, L. Xiao. Chem. Eng. Technol. 2010. 33, 2, 244.

14. D.V. Bavykin, A.A. Lapkin, P.K. Plucinski, J.M. Friedrich, F.C. Walsh. J. Phys. Chem. B. 2005. 109, 41, 19422. 
15. L. Torrente-Murciano, A.A. Lapkin, D.V. Bavykin, F.C. Walsh, K. Wilson. J. Catalysis. 2007. 245, 2, 272.

16. L. Yu, X. Zhang. Mat. Chem. Phys. 2004. 87, 1, 168.

17. Z.-R. Tang, X. Yin, Y. Zhang, Y.-J. Xu. Inorg. Chem. 2013. 52, 20, 11758.

18. C. Huang, X. Liu, L. Kong, W. Lan, Q. Su, Y. Wang. Appl. Phys. A. 2007. 87, 781.

19. A.-L. Papa, L. Dumont, D. Vandroux, N. Millot. Nanotoxicology. 2013. 7, 6, 1131.

20. H.-J. Song, Z.-Z. Zhang, X.-H. Men. Eur. Polym. J. 2008. 44, 4, 1012.

21. L. Torrente-Murciano, A.A. Lapkin, D. Chadwick. J. Mater. Chem. 2010. 20, 6484.

22. P. Herrasti, A.N. Kulak, D.V. Bavykin, C. Ponce de Léon, J. Zekonyte, F.C. Walsh. Electrochim. Acta. 2011. 56, 1323.

23 Xiaoming Fan; Khosravi, F.; Rahneshin, V.; Shanmugam, M.; Loeian, M.; Jasinski, J.;

Cohn, R.W.; Terentjev, E.; Panchapakesan, B., Nanotechnology Vol: 26 (26) 2015 Page: 261001 (11 pp.).

24 C. Dechakiatkrai, C. Lynam, K.J. Gilmore, J. Chen, S. Phanichphant, D.V. Bavykin, F.C. Walsh, G.G. Wallace, "Single-walled Carbon Nanotube/Titanate Nanotube Composite Fibres", Advanced Engineering Materials, 2009, 11, B55-B60.

25. E. Kymakis, I. Alexandrou, G.A.J. Amaratunga. Synthetic metals. 2002. 127, 59.

26. H. Kim, A.A. Abdala, C.W. Macosko. Macromolecules. 2010. 43, 16, 6515.

27. H. Hu, X. Wang, J. Wang, L. Wan, F. Liu, H. Zheng, R. Chen, C. Xu. Chem. Phys. Lett. 2010. 484, 247.

28 Y. Gao, X. Gao, Y. Zhou, D. Yan, Nanotechnology, 19, (2008) 495604

29 F.C. Walsh, C. Ponce de León, Trans. Inst. Mats Finish., 2014, 92, 83.

30. M. Rinaudo. Prog. Polym. Sci. 2006. 31, 7, 603.

31. R. Jayakumar, M. Prabaharan, S.V. Nair, H. Tamur. Biotech. Adv. 2010. 28, 1, 142. 
32. A.R Bhattacharyya, T.V. Sreekumar, T. Liu, S. Kumar, L.M. Ericson, R.H. Hauge, R.E. Smalley. Polymer. 2003. 44, 8, 2373.

33. Y.T. Shah, A.B. Pandit, V.S. Moholkar. Cavitation reaction engineering. Kluwer Academic/Plenum Publishers. 1999.

34. D.V. Bavykin, B.A. Cressey, M.E. Light, F.C. Walsh. Nanotechnology. 2008, 19, 27, 275604.

35. B.D. Beake, S. Chen, J.B. Hull, F. Gao. J. Nanosci. Nanotech. 2002, 2, 1, 73.

36. W.C. Oliver, G.M. Pharr. J. Mater. Res. 1992, 6, 1564.

37. F. Hennrich, R. Krupke, K. Arnold, J.A.R. Stutz, S. Lebedkin, T. Koch, T. Schimmel, M.M. Kappes. J. Phys. Chem. B. 2007, 111, 1932.

38. J. Hilding, E.A. Grulke, Z.G. Zhang, F. Lockwood. J. Disp. Sci. Tech. 2003, 24, 1.

39. A. Lucas, C. Zakri, M. Maugey, M. Pasquali, P. van der Schoot, P. Poulin. J. Phys. Chem. C. 2009, 113, 20599.

40. G. Pagani, M.J. Green, P. Poulin, M. Pasquali. PNAS. 2012, 109, 29, 11599.

41. G. Kozma, Z. Konya, A. Kukovecz, RSC Advances, 2013, 3, 21, 7681.

42. S.A. Vanapalli, S. L. Ceccio, M. J. Solomon. PNAS. 2006, 103, 45, 16660.

43. D. Qian, E.C. Dickey, R. Andrews, T. Rantell. Appl. Phys. Lett. 2000, 76, 2868.

44. B. Arash, Q. Wang, V. K. Varadan. Scientific Reports. 2014, 4, 6479. 


\title{
Titanate Nanotubes for Reinforcement of a Poly(Ethylene Oxide)/Chitosan Polymer Matrix
}

\author{
R. Porras ${ }^{\mathrm{a}}$, D.V. Bavykin ${ }^{\mathrm{a}}$, J. Zekonyte ${ }^{\mathrm{b}}$, F.C. Walsh ${ }^{\mathrm{a}}$, R.J. Wood ${ }^{\mathrm{b}}$ \\ ${ }^{a}$ Energy Technology Research Group, Engineering Sciences, Faculty of Engineering and the \\ Environment, University of Southampton, UK \\ ${ }^{b}$ National Centre for Advanced Tribology, Engineering Sciences, Faculty of Engineering and \\ the Environment, University of Southampton, UK \\ Supplementary information
}




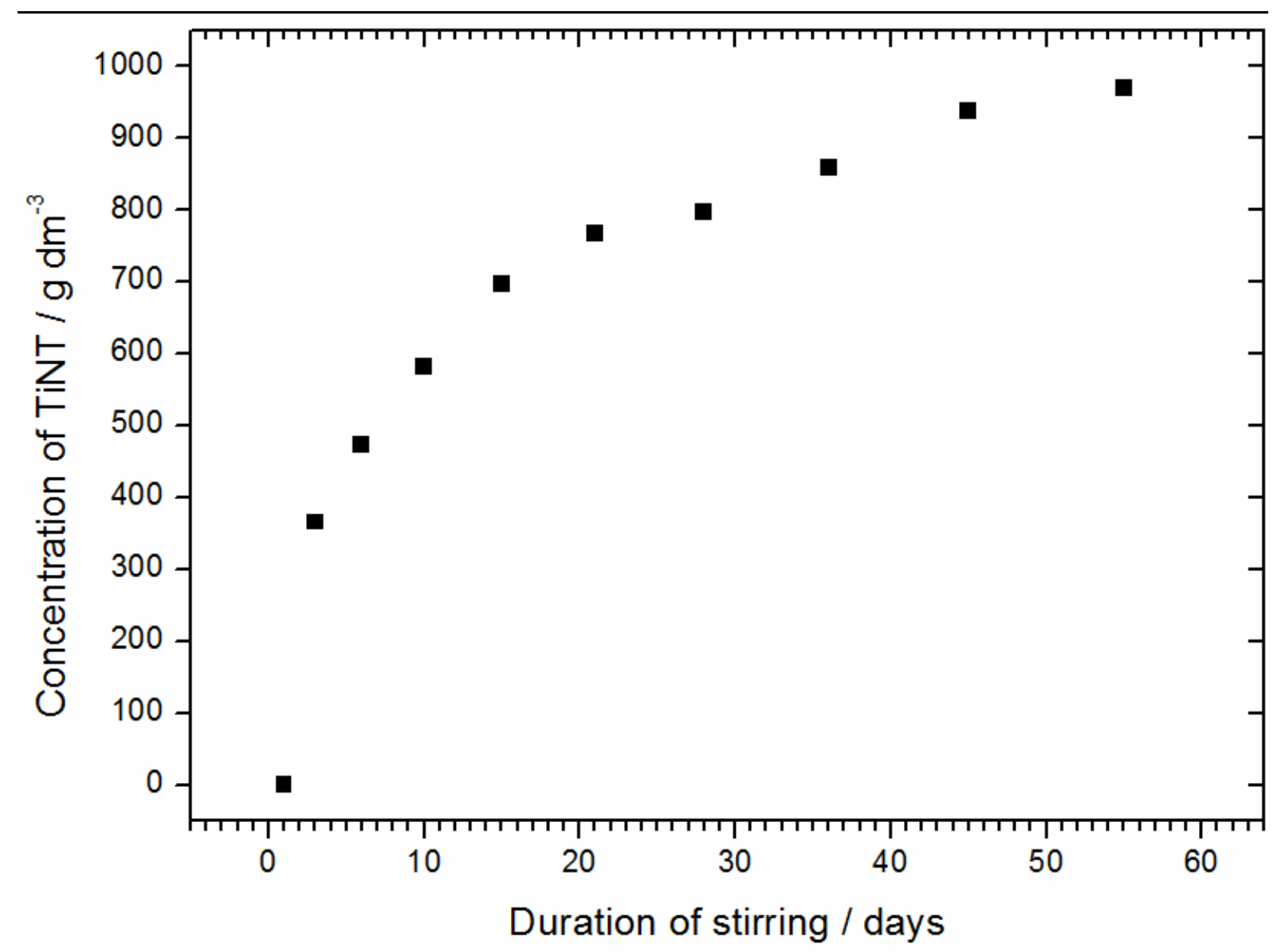

Figure S1. Kinetic curve of accumulation of isolated TiNT in the water during mechanical stirring of the $400 \mathrm{mg}$ of nanotubes suspended in $200 \mathrm{dm}^{3}$ of water. Stirred using a cylindrical PTFE coated steel stirring bar $20 \mathrm{~mm}$ long and $5 \mathrm{~mm}$ diameter at a rotation rate of $700 \mathrm{rpm}$.

Table S1. Hardness and reduced Young's modulus of composite PEO:CS (6:1) films produced with controlled TiNT content.

\begin{tabular}{ccc}
\hline Composite Film & $H / \mathrm{GPa}$ & $\mathrm{Er} / \mathrm{GPa}$ \\
\hline PEO:CS 6:1 & $0.073 \pm 0.008$ & $1.948 \pm 0.162$ \\
PEO:CS 6:1 + 3\% TiNT & $0.120 \pm 0.020$ & $3.551 \pm 0.504$ \\
PEO:CS 6: $1+6 \%$ TiNT & $0.150 \pm 0.018$ & $3.891 \pm 0.494$ \\
PEO:CS 6:1 + 9\% TiNT & $0.139 \pm 0.022$ & $3.505 \pm 0.548$ \\
PEO:CS 6:1+ 12.5\% TiNT & $0.159 \pm 0.073$ & $4.193 \pm 1.446$ \\
PEO:CS 6: $1+15 \%$ TiNT & $0.141 \pm 0.031$ & $3.725 \pm 0.763$ \\
PEO:CS 6:1 + 20\% TiNT & $0.129 \pm 0.017$ & $3.102 \pm 0.416$ \\
PEO:CS 6:1 + 25\% TiNT & $0.192 \pm 0.021$ & $6.646 \pm 0.729$ \\
PEO:CS 6: $1+30 \%$ TiNT & $0.180 \pm 0.015$ & $5.218 \pm 0.479$ \\
\hline
\end{tabular}


Table S2. Hardness and reduced Young's modulus of PEO:CS 6:1 TiNT $4.7 \mathrm{wt} \%$ composite films before and after ultrasound treatment.

\begin{tabular}{ccc}
\hline Composite Film & $H / \mathrm{GPa}$ & $\mathrm{Er} / \mathrm{GPa}$ \\
\hline PEO:CS 6:1 & $0.073 \pm 0.008$ & $1.948 \pm 0.162$ \\
$4.7 \%$ TiNT No Usound & $0.109 \pm 0.019$ & $2.892 \pm 0.399$ \\
4.7\% TiNT 15 min & $0.124 \pm 0.029$ & $2.358 \pm 0.479$ \\
4.7\% TiNT 30 min & $0.146 \pm 0.038$ & $3.683 \pm 0.742$ \\
4.7\% TiNT 1 h & $0.136 \pm 0.054$ & $3.973 \pm 1.202$ \\
4.7\% TiNT 2 h & $0.095 \pm 0.020$ & $2.433 \pm 0.404$ \\
4.7\% TiNT 3 h & $0.126 \pm 0.059$ & $1.388 \pm 0.419$ \\
\hline
\end{tabular}

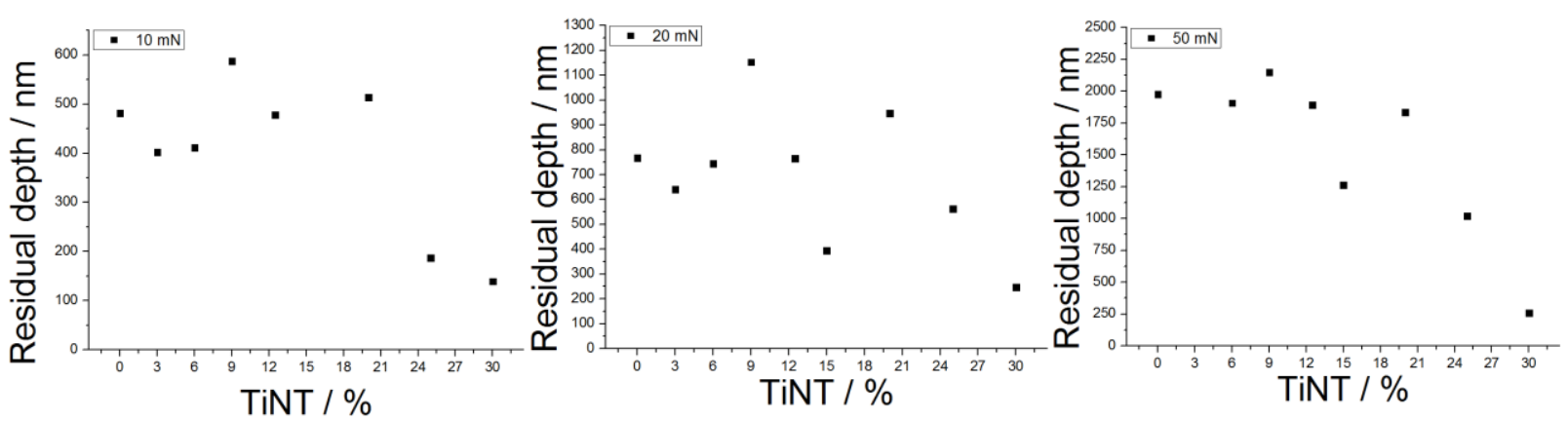

Figure S2 Nanoindentation residual depth $v s$. TiNT wt $\%$ in composite films under 10, 20 and $50 \mathrm{mN}$ applied loads.
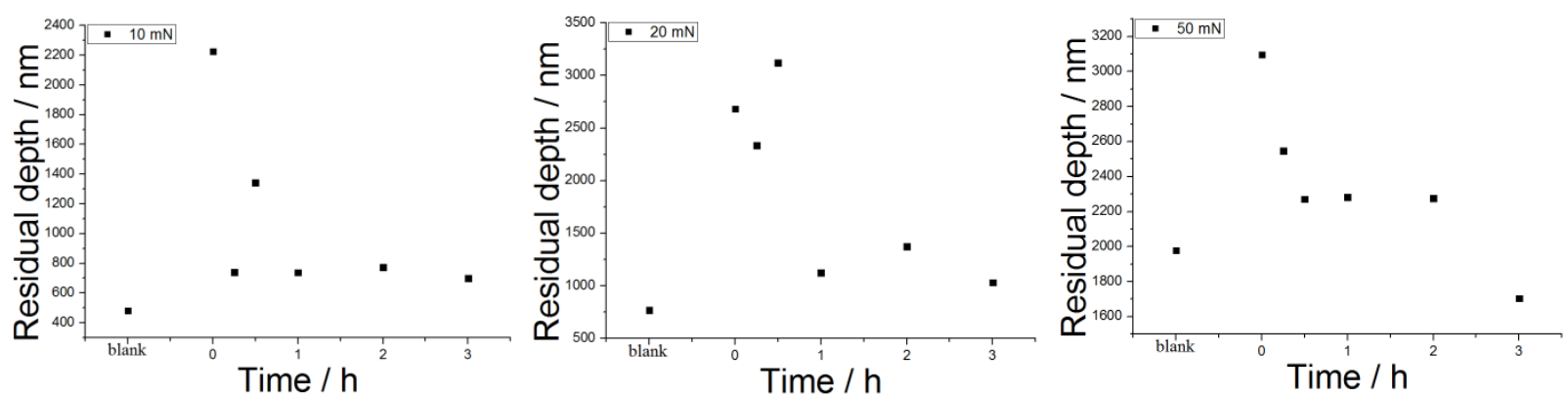

Figure S3. Nanoindentation residual depth $v s$. ultrasound treatment time of PEO:CS 6:1 TiNT $4.7 \mathrm{wt} \%$ composite films under 10, 20 and $50 \mathrm{mN}$ applied loads. 


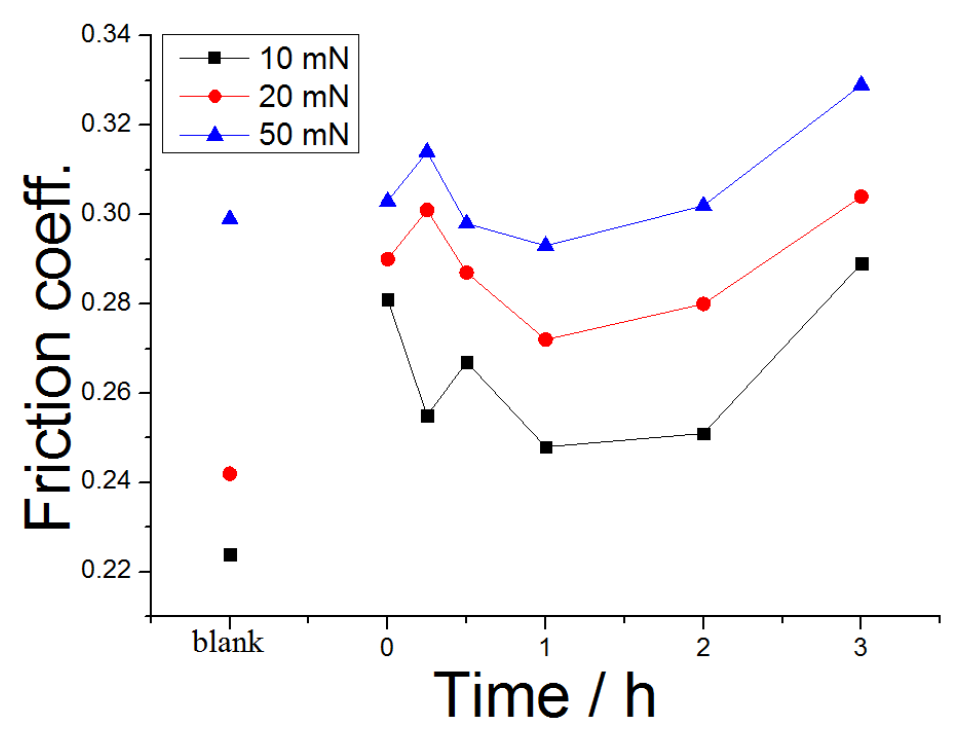

Figure S4. Friction coefficient vs. ultrasound treatment. The first data points correspond to a neat polymer blend of 6:1 PEO:CS. 